Impact of pressure anisotropy on magnetic configuration and stability

This content has been downloaded from IOPscience. Please scroll down to see the full text. 2016 Nucl. Fusion 56112017

(http://iopscience.iop.org/0029-5515/56/11/112017)

View the table of contents for this issue, or go to the journal homepage for more

Download details:

IP Address: 130.56 .106 .27

This content was downloaded on 31/03/2017 at 06:26

Please note that terms and conditions apply.

You may also be interested in:

Resolving the wave-particle-plasma interaction: advances in the diagnosis, interpretation and self-consistent modelling of waves, particles and the plasma configuration

$\mathrm{M} J$ Hole and M Fitzgerald

Fast particle modifications to equilibria and resulting changes to Alfvén wave modes in tokamaks M J Hole, G von Nessi, M Fitzgerald et al.

Modeling the effect of anisotropic pressure on tokamak plasmas normal modes and continuum using fluid approaches

Z S Qu, M J Hole and M Fitzgerald

Energetic particle physics in fusion research in preparation for burning plasma experiments

N.N. Gorelenkov, S.D. Pinches and K. Toi

Internal amplitude of frequency sweeping TAE

S D Pinches, H L Berk, M P Gryaznevich et al.

Saturation of Alfvén modes in tokamak plasmas investigated by Hamiltonian mapping techniques

S. Briguglio, M. Schneller, X. Wang et al.

Energetic particles in spherical tokamak plasmas

K G McClements and E D Fredrickson

Analysing the impact of anisotropy pressure on tokamak equilibria

ZS Qu, M Fitzgerald and M J Hole 


\title{
Impact of pressure anisotropy on magnetic configuration and stability
}

\author{
B. Layden ${ }^{1}$, Z.S. Qu ${ }^{1}$, M. Fitzgerald ${ }^{2}$ and M.J. Hole ${ }^{1}$ \\ ${ }^{1}$ Research School of Physics and Engineering, The Australian National University, \\ Canberra ACT 2601, Australia \\ ${ }^{2}$ CCFE, Culham Science Centre, Abingdon, Oxon, OX14 3DB, UK \\ E-mail: brett.layden@anu.edu.au
}

Received 8 March 2016, revised 17 June 2016

Accepted for publication 30 June 2016

Published 3 August 2016

\begin{abstract}
A number of tools have recently been developed to study equilibrium and stability in tokamaks with pressure anisotropy. Here we apply these tools to a Mega Ampere Spherical Tokamak (MAST) discharge to calculate equilibrium, Alfvén continua and eigenmodes, through to linear growth and nonlinear saturation of a toroidal Alfvén eigenmode (TAE); this is the first study of wave growth and saturation for anisotropic equilibria. Comparisons with the standard tools which assume an isotropic pressure reveal various differences in equilibrium and modes: the safety factor profile in the isotropic reconstruction is reversed shear while the anisotropic reconstruction gives normal shear; the isotropic TAE gap is much narrower than the anisotropic gap; and the TAE radial mode structure is wider in the anisotropic case. These lead to a modification in the resonant regions of fast-ion phase space, and produce a $35 \%$ larger linear growth rate and an $18 \%$ smaller saturation amplitude for the TAE in the anisotropic analysis compared to the isotropic analysis.
\end{abstract}

Keywords: tokamak, equilibrium, stability, anisotropy, MHD, energetic particles, MAST

(Some figures may appear in colour only in the online journal)

\section{Introduction}

External heating in modern tokamaks can lead to momentum injection and velocity-space or pressure anisotropy. Such physics can displace flux surfaces outwards, and lead to additional currents that can modify the magnetic configuration and change plasma wave modes and stability. For instance, for values of $p_{\perp} / p_{\|} \approx 1.5, p_{\perp}$ can vary by $20 \%$ at mid-radius for a spherical tokamak equilibrium [1]. A number of authors report that the experimentally inferred equilibrium, and in particular the on-axis safety factor, can be significantly different if a single pressure is assumed rather than anisotropic pressures [2-4]. Qu et al [1] find that these differences increase with increasing anisotropy and inverse aspect ratio. At high beta the impact of anisotropy is non-perturbative: recent work [5] has shown that at very high beta the impact of non-zero anisotropy is to eliminate the diamagnetic hole that would otherwise be present in isotropic plasmas [6,7], even with flow [8]. Further discussion on the impact of anisotropy can be found in the contemporary topical reviews by Pustovitov [9] and Hole and Fitzgerald [10].
In recent years a range of new tools has been developed to model high-performance plasmas and understand the change in plasma wave mode and stability. EFIT TENSOR [11] is a modification of the existing force-balance solver EFIT ++ , which is constrained to external magnetic measurements and temperature and density profiles, together with user-input constraints of on-axis safety factor. EFIT TENSOR adds kinetic constraints $p_{\|}$and $p_{\perp}$ and a toroidal flow profile. HELENA + ATF [1] is a fixed boundary solver that includes anisotropy and toroidal flow, and computes the field configuration in the metric used by MHD stability code MISHKA [12]. We have also developed a new single adiabatic stability theory for anisotropic plasma that reverts to MHD in the isotropic limit [13], and implemented this and double-adiabatic closure in the extended MHD stability code MISHKA-A [14]. In tandem to these developments we have also deployed Bayesian inference techniques to infer both the toroidal and poloidal rotation profiles [3] and energetic particle pressure [4, 15].

In this work we apply these advances in theory and computational models to study mode activity in the UK Mega 


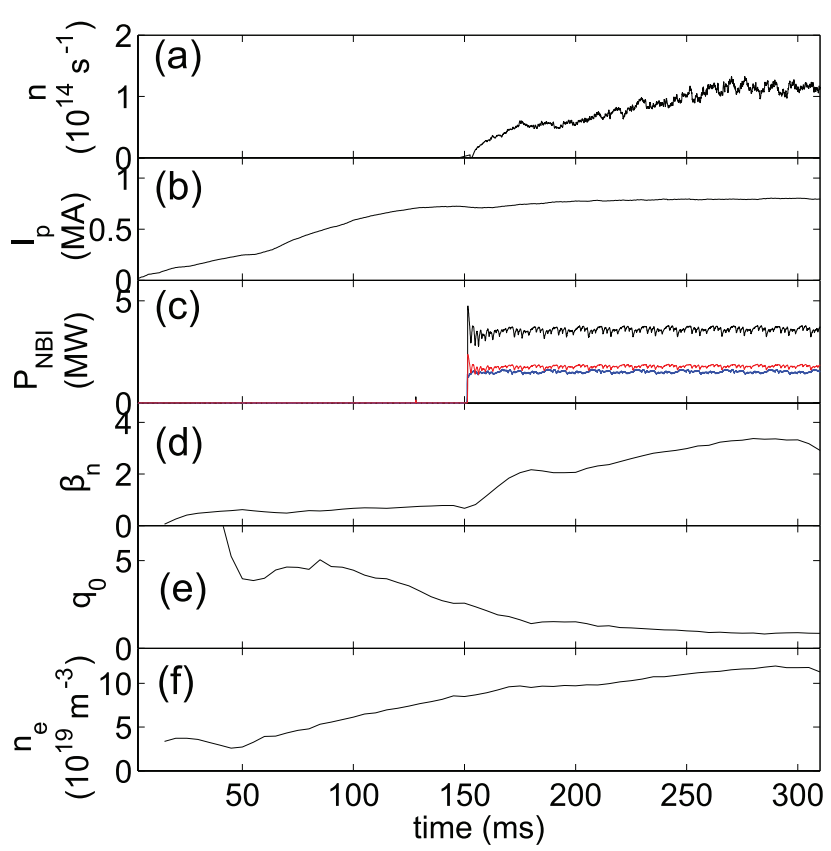

Figure 1. Evolution of discharge \#29221. Figure (a) shows neutron rate, $(b)$ plasma current $I_{\mathrm{p}},(c)$ neutral beam power $P_{\mathrm{NBI}}$ (blue is the SS supercusp beam, red is the SW chequerboard beam, and black is the total), $(d)$ the normalised beta $\beta_{n},(e)$ the core safety factor $q_{0},(f)$ the line integrated electron density $n_{\mathrm{e}}$. The quantities $q_{0}$ and $\beta_{n}$ are inferred quantities computed using EFIT.

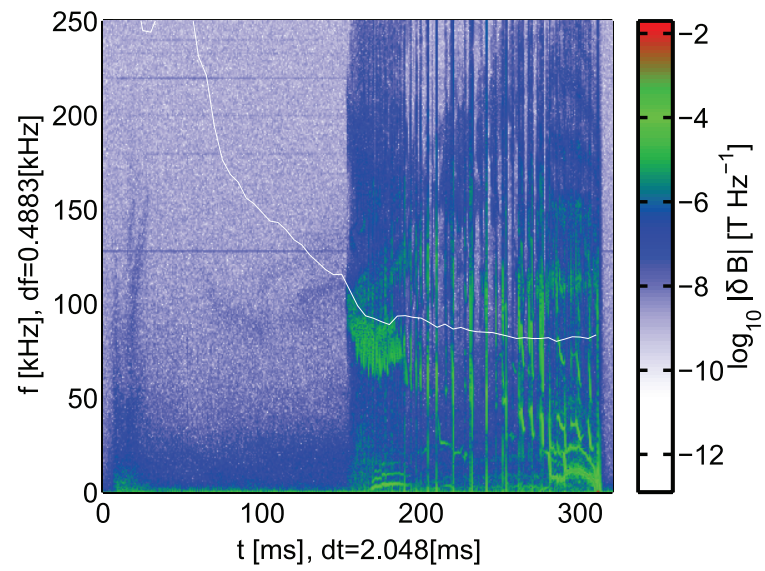

Figure 2. Spectrogram of discharge \#29221. The white trace is the TAE frequency, $v_{A} / 2 q_{0} R$, using the $q_{0}$ value in figure $1(e)$.

Ampere Spherical Tokamak (MAST). MAST discharge \#29221, produced during a power-density scan set of experiments [16], was a 3.1 MW two-beam heated plasma (a southsouth (SS) 'supercusp' beam operating at 1.5 MW and a south-west (SW) 'chequerboard' beam operating at 1.6 MW) with a plasma current of $0.9 \mathrm{MA}$ and normalised beta $\beta_{n} \approx 3$. Figure 1 is time trace of the evolution of the discharge, and figure 2 a spectrogram of magnetic perturbation coil data. The magnetics reveal a rich range of activity, including: suspected low-frequency $(10 \mathrm{kHz})$ tearing modes at $170 \mathrm{~ms}$; toroidal Alfvén eigenmodes (TAEs) from $150 \mathrm{~ms}$ to $220 \mathrm{~ms}$ with frequency ramping down from $100 \mathrm{kHz}$ to $50 \mathrm{kHz}$; chirping-down fishbones from $250 \mathrm{~ms}$ to $300 \mathrm{~ms}$; and beyond $280 \mathrm{~ms}$, longlived mode activity. Keeling et al [16] focused on the fishbone

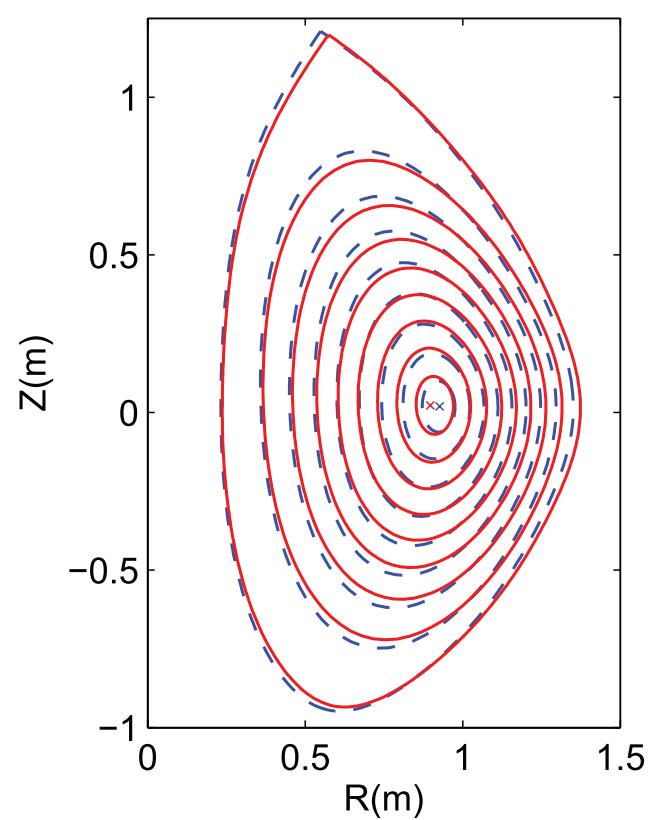

Figure 3. Flux surfaces for the isotropic (red solid line) and anisotropic (blue dashed line) equilibrium reconstructions, with the crosses showing the location of the respective magnetic axes.

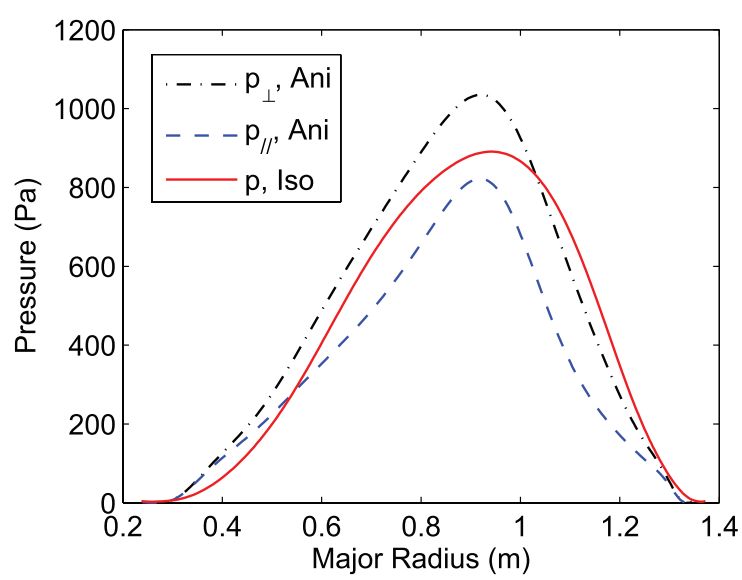

Figure 4. The pressure profiles along the midplane for the isotropic and anisotropic equilibria.

phase just prior to long-lived mode activity. Our focus is the TAE mode activity at $190 \mathrm{~ms}$, where a high fidelity TRANSP simulation is available. At this time the observed mode frequency is $\approx 70 \mathrm{kHz}$, with a frequency chirp of $20 \mathrm{kHz}$ across a $2 \mathrm{~ms}$ slice $(\approx 80 \mathrm{kHz}$ mode frequency at $189 \mathrm{~ms}$ and $\approx 60 \mathrm{kHz}$ at $191 \mathrm{~ms}$ ). Toroidal mode number analysis shows that $n=1$ for these modes.

\section{Equilibrium and mode calculation}

In this work, we examine two equilibrium reconstructions for MAST \#29221 using different models and assumptions. The first 'anisotropic' equilibrium is reconstructed by EFIT TENSOR, from experimental constraints such as the external coils, the total current, the Motional Stark Effect (MSE) spectroscopy, and kinetic constraints $\left(p_{\|}\right.$and $\left.p_{\perp}\right)$ from TRANSP simulations with an empirical fast particle diffusivity that 

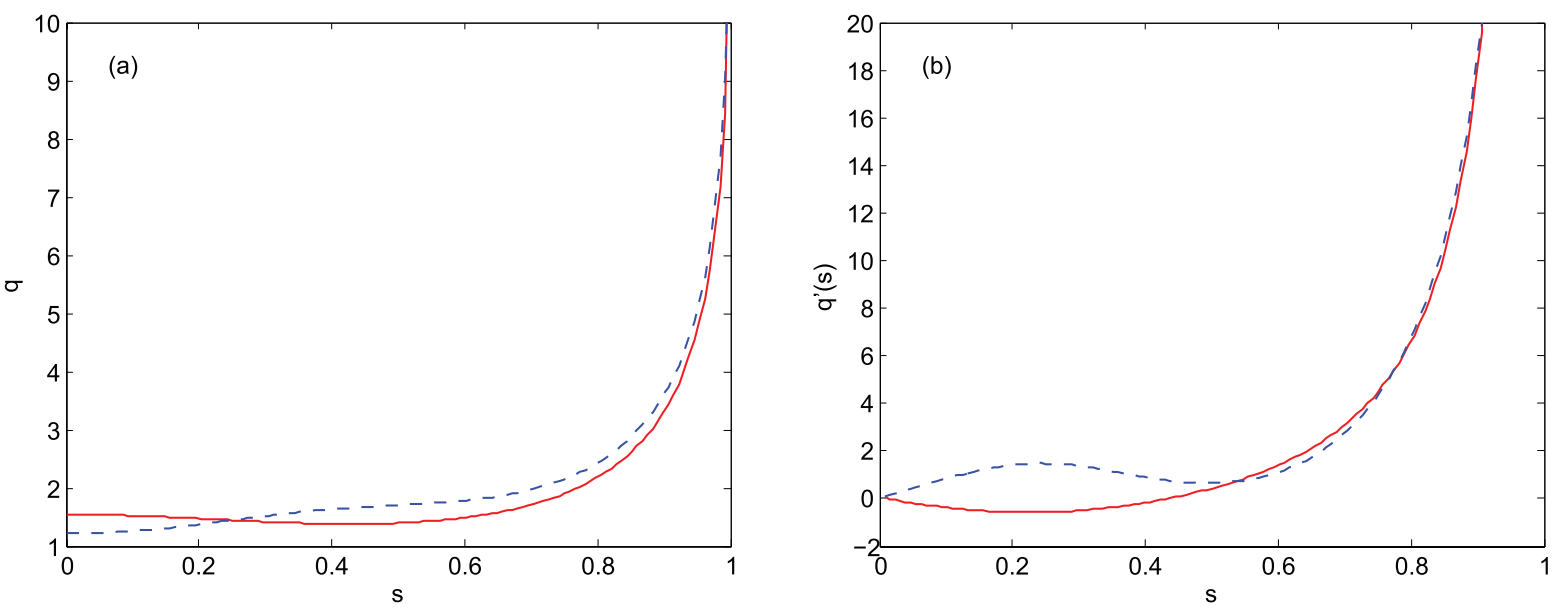

Figure 5. (a) The $q$ profile and $(b) q^{\prime}(s)$ for the isotropic equilibrium (red solid line) and anisotropic equilibrium (blue dashed line).
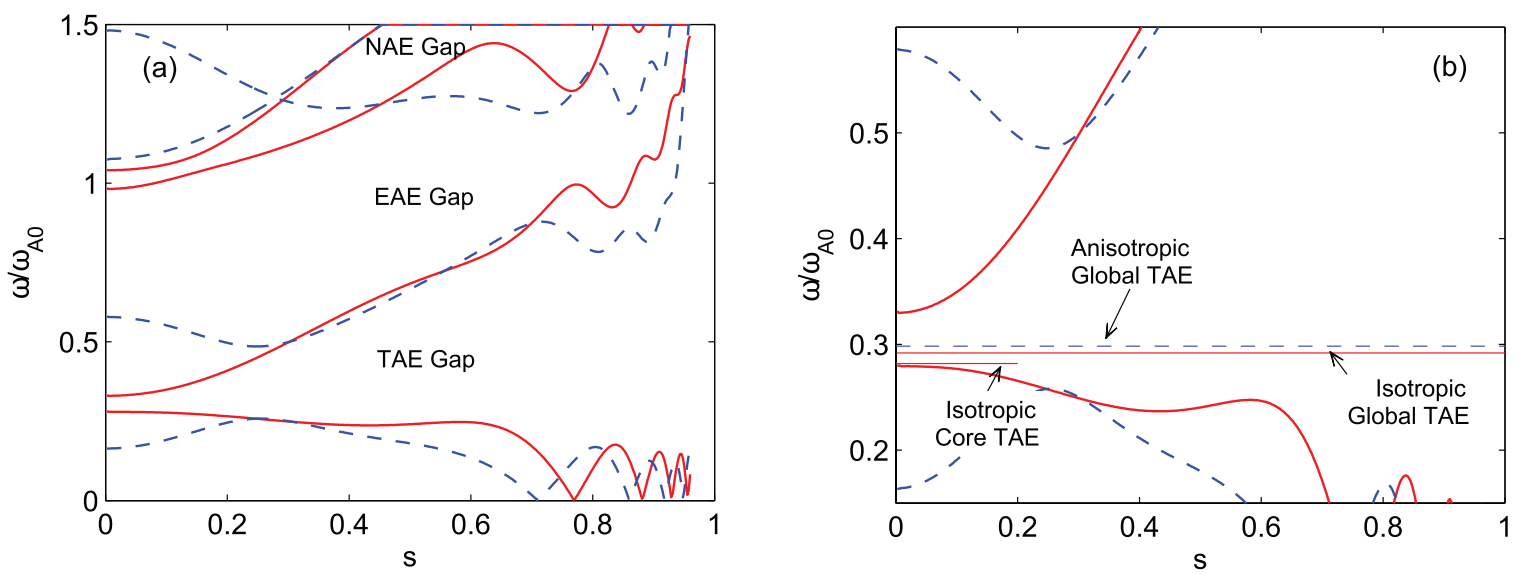

Figure 6. The $n=1, m=1-11$ incompressible continuous spectrum and the TAE frequencies of the isotropic equilibrium (red solid line) and anisotropic equilibrium (blue dashed line), where the frequency $\omega$ is normalised by the on-axis Alfvén frequency $\omega_{\mathrm{A} 0}$, and $s=\sqrt{\psi / \psi_{\text {edge }}}$ is a normalised poloidal flux coordinate. Figure 6(a) shows the spectrum over a large frequency range with the TAE, EAE, and NAE gaps labelled, and figure $6(b)$ shows a magnification of the TAE-gap frequency range, with the horizontal lines representing the TAE frequencies found by MISHKA(-A).

provided a match to the measurements of neutron diagnostics. The second 'isotropic' equilibrium is reconstructed by EFIT ++ (isotropic) with the same constraints, except that the flux-surface averaged $\left\langle p^{*}\right\rangle$, where $p^{*} \equiv\left(p_{\|}+p_{\perp}\right) / 2$, is used as the kinetic constraint. The isotropic and anisotropic flux surfaces are shown in figure 3. Both reconstructions give strongly shaped flux surfaces with an $\mathrm{X}$-point at $R \sim 0.55 \mathrm{~m}$ and $Z \sim 1.2 \mathrm{~m}$; there is a small relative displacement between the isotropic and anisotropic flux surfaces and magnetic axes. The pressure profiles are plotted in figure 4 as a function of major radius along the midplane $\left(Z=Z_{\mathrm{mag}}\right)$. It is interesting to note that although both cases are constrained to MSE, the $q$ profiles are substantially different, as shown in figure 5; here $q$ is graphed as a function of the normalised poloidal flux coordinate $s \equiv \sqrt{\psi / \psi_{\text {edge }}}$. The $q$ profile in the isotropic equilibrium is reversed shear, while for the anisotropic equilibrium it is normal shear with a 'dip' on-axis. Finally, we note that both $q$ profiles have a flat region for $s<0.6$.

The EFIT ++ (EFIT TENSOR) flux functions and the last closed flux surface are used as inputs to the fixed-boundary equilibrium solver HELENA (HELENA+ATF) to obtain the metrics in straight-field-line coordinates for the isotropic (anisotropic) equilibrium. The continuous spectra of the isotropic and anisotropic equilibria are then calculated by CSMISH [17] and CSMISH-A [14], respectively, each using the density profile $n(s)=n_{0}\left(1-0.7 \mathrm{~s}^{2}\right)$ as a reasonable fit to the Thomson scattering data, with $n_{0}=2.42 \times 10^{19} \mathrm{~m}^{-3}$ the on-axis number density. Figure $6(a)$ is an overview of the $n=1$ incompressible continuum for each equilibrium. Three gaps (TAE, EAE, and NAE, respectively induced by the toroidicity, ellipticity, and triangularity) exist in the frequency range $0 \leqslant \omega \leqslant 1.5 \omega_{\mathrm{A} 0}$ where $\omega_{\mathrm{A} 0}=B_{0} / R_{0} \sqrt{\mu_{0} \rho_{0}}$ is the Alfvén frequency at the magnetic axis. The TAE gap of the isotropic equilibrium is much narrower than that of the anisotropic equilibrium, due mainly to the difference in the $q$ profile shown in figure 5 . The $m=1$ and $m=2$ continua intersect at the $q=1.5$ surface which is located at $s \approx 0.3$ for the anisotropic equilibrium. However, for the isotropic equilibrium, two $q=1.5$ surfaces exist (at $s=0.15$ and $s=0.5$ ) due to the reverse shear. The intersection at $s=0.15$ will create a much 

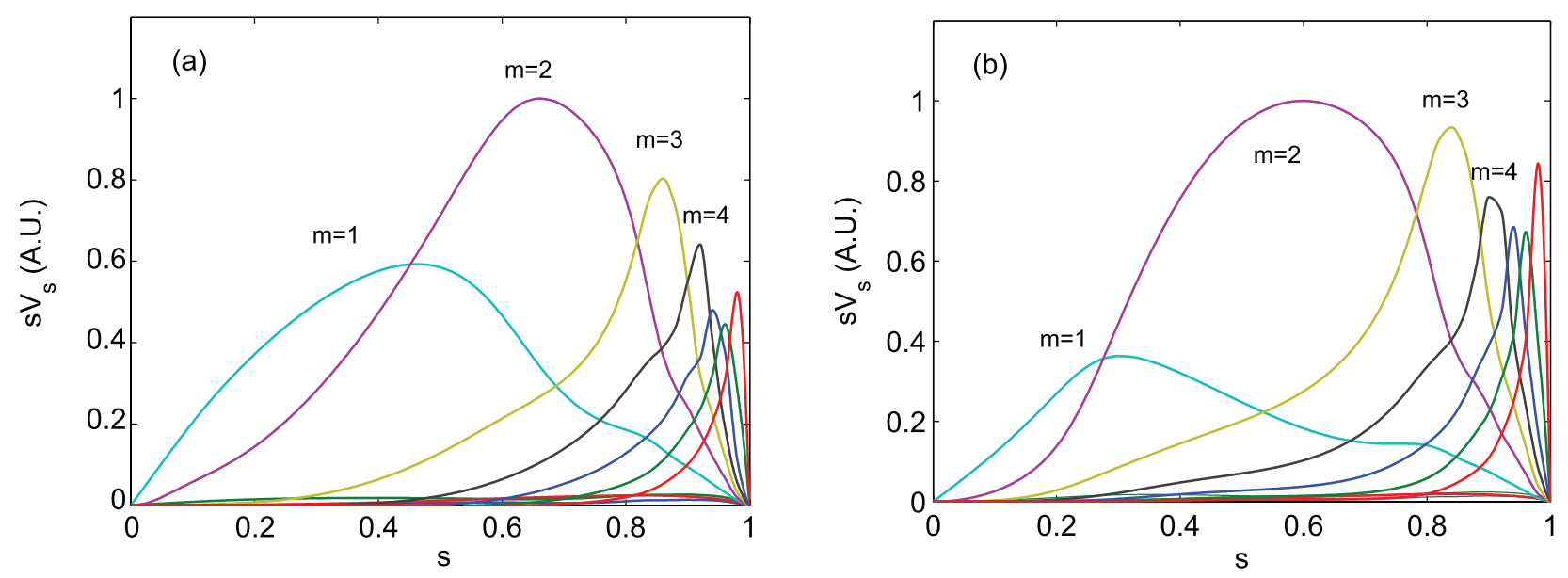

Figure 7. The $n=1$ global TAE radial structure of $(a)$ the isotropic equilibrium and $(b)$ the anisotropic equilibrium.

narrower gap, since the inverse aspect ratio $\epsilon=r / R$ is smaller and thus the toroidicity effects are weaker. Finally, we note that the EAE and NAE gaps are closed in both equilibria.

The continuum gaps allow the existence of robust global modes that are free from continuum damping. In this work, we will focus on $n=1$ TAEs that are observed on the Mirnov coil spectrogram. The corresponding TAE gap is shown in figure 6(b). We use MISHKA and MISHKA-A to calculate the TAEs for the isotropic and anisotropic equilibria, respectively. Two TAEs are found for the isotropic case due to the reverse shear, one of which is core localised $(s<0.2)$ and therefore less likely to be picked up by the external coils. The frequency of this core mode is $78.2 \mathrm{kHz}$, just $0.1 \mathrm{kHz}$ above the lower continuum tips. The other, however, is a global mode with a frequency of $81.3 \mathrm{kHz}$, with the radial mode structure shown in figure $7(a)$. For the anisotropic equilibrium, a global mode is also found at $88.9 \mathrm{kHz}$. The radial mode structure, especially the $m=2$ harmonic, is broader compared to the isotropic one, as seen in figure $7(b)$.

\section{Stability}

\subsection{Physical background and method}

Alfvén waves can be driven unstable by fast ions produced by neutral beam injection. For shear Alfvén waves, which are transverse electromagnetic waves, the drift motion of the fast ions gives the dominant contribution to the wave-particle interaction [18]. The power transfer between fast ions and shear Alfvén waves is approximately proportional to $\boldsymbol{v}_{d} \cdot \boldsymbol{E}_{\perp}$, with $\boldsymbol{v}_{d}$ the drift velocity and $\boldsymbol{E}_{\perp}$ the transverse electric field. Net energy transfer therefore requires that $\boldsymbol{v}_{d} \cdot \boldsymbol{E}_{\perp}$ averaged over many periods be non-zero, leading to the resonance condition [18-20]

$$
\Omega_{n, l}=\omega+l \omega_{\theta}-n \omega_{\phi}=0,
$$

where $n$ is the toroidal mode number, $l$ is an integer, $\omega$ is the angular wave frequency, and $\omega_{\theta}$ and $\omega_{\phi}$ are respectively the poloidal and toroidal orbit frequencies.

The energy transfer between waves and particles depends on a number of factors [18]. The first is the relative amplitudes of the poloidal harmonics of the eigenmode, which will affect the strength of the different resonances. The growth rate $\gamma$ of the wave amplitude depends on the slope of the distribution function at resonance through [18]

$$
\gamma \propto \omega \frac{\partial f}{\partial E}+n \frac{\partial f}{\partial P_{\phi}}
$$

where $P_{\phi}=m R v_{\phi}-Z e \psi$ is the toroidal angular momentum. Since $P_{\phi}$ increases as $\psi$ decreases, a negative gradient $\partial f / \partial \psi$ drives wave growth. Energy transfer is also dependent on the alignment of the particle orbit and eigenmode. The energy transfer is maximised when the drift-orbit width $\Delta_{b}$ is comparable to the eigenmode width $\Delta_{m}[18,21,22]$. In the case where these widths are not comparable, large orbit-width theory $\left(\Delta_{b} \gg \Delta_{m}\right)$ predicts a reduced power transfer than that obtained in narrow-orbit width theory $\left(\Delta_{b} \ll \Delta_{m}\right)[23,24]$.

A quantitative treatment of the wave-particle energy transfer requires a numerical solution of the assumed model for the wave-particle interaction. The HAGIS code [25] is a nonlinear perturbative code that solves the drift-kinetic equation in toroidal geometry for a distribution of fast particles and a set of Alfvén eigenmodes. The fast particle motion is described in HAGIS by a guiding-centre Hamiltonian in Boozer coordinates, with the assumption of isotropic bulk plasma pressure.

A rigorous approach to modelling the wave-particle interaction in plasmas with pressure anisotropy requires the use of an anisotropic Hamiltonian. A guiding-centre Hamiltonian in Boozer coordinates for plasmas with pressure anisotropy has been derived in $[26,27]$. Unlike in the isotropic case, it is the lines of effective current density $\boldsymbol{K} \equiv \nabla \times(\sigma \boldsymbol{B}) / \mu_{0}$ (with $\left.\sigma \equiv 1-\mu_{0}\left(p_{\|}-p_{\perp}\right) / B^{2}\right)$ which lie on flux surfaces [28, 29]; that is, $\boldsymbol{K} \cdot \nabla \psi=0$ rather than $\boldsymbol{J} \cdot \nabla \psi=0$, where $\boldsymbol{J}$ is the true current density. From the conditions $\boldsymbol{K} \cdot \nabla \psi=0$ and $\nabla \cdot \boldsymbol{K}=0$, the magnetic field can be expressed in the covariant Boozer representation as $\sigma \boldsymbol{B}=g(\psi) \nabla \phi+I(\psi) \nabla \theta+g(\psi) \delta(\psi, \theta) \nabla \psi$. This modifies the expressions for the canonical momenta relative to the isotropic case, and thus changes the equations of motion. The rigorous approach to modelling the wave-particle interaction with pressure anisotropy would involve implementing these new equations of motion into HAGIS. 

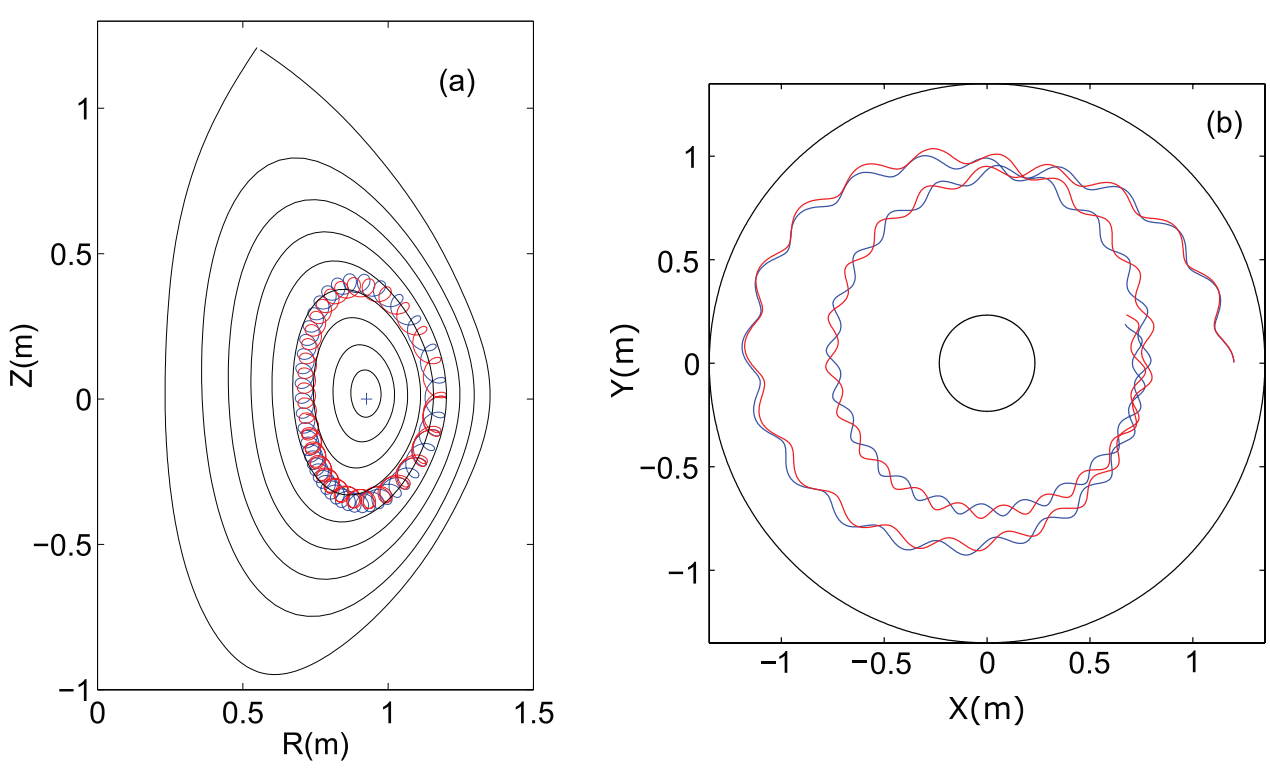

Figure 8. Fast ion orbits calculated using CUEBIT for the full anisotropic equilibrium (blue) and the remapped equilibrium (red) for MAST \#29221 for ions with $E=21 \mathrm{keV}$ and $\Lambda=0.47$, where $(a)$ and $(b)$ show the poloidal projection and top view of the particle orbits respectively.

In this paper, however, we propose an approximate method for the inclusion of pressure anisotropy in wave-particle interaction studies without the need to modify the HAGIS code. First, the anisotropic equilibrium is computed using HELENA + ATF. Flux surface averages are then calculated for the toroidal current $J_{\phi}$ and for $p^{*} \equiv\left(p_{\|}+p_{\perp}\right) / 2$. We then input these $\left\langle J_{\phi}\right\rangle(\psi)$ and $\left\langle p^{*}\right\rangle(\psi)$ into the standard HELENA code (with $\left\langle p^{*}\right\rangle$ taken as the 'isotropic' pressure), applying the same boundary conditions as in HELENA + ATF, and rescale the total current such that the $q$-profile matches that obtained from HELENA + ATF. The use of this remapped equilibrium and isotropic Hamiltonian is appropriate if the particle orbits match those for the fully anisotropic equilibrium and anisotropic Hamiltonian.

Here, to assess the validity of the approximate approach, we have calculated trapped and passing orbits using the fullorbit code CUEBIT [30] for MAST \#29221 for both the full anisotropic and remapped equilibria. We show an example of a co-passing orbit in figure 8 for a given particle energy $E$ and orbit-invariant $\Lambda \equiv \mu B_{0} / E$. We find that the poloidal orbit frequencies of the passing particle in the full anisotropic and remapped cases are $118.6 \mathrm{kHz}$ and $119.4 \mathrm{kHz}$ respectively, so the remapped orbit frequency is less than $1 \%$ larger than the anisotropic orbit frequency. In addition, we see from figure 8 that the guiding-centre particle trajectories are similar. Since the particles have the same initial value of major radius ( $\left.R=R_{\max }\right)$ along the equatorial plane $\left(Z=Z_{\text {mag }}\right)$ on the lowfield side, the difference between these particle orbits is largest on the equatorial plane on the high-field side where $R=R_{\min }$. We find that the difference in $R_{\max }-R_{\min }$ is approximately $1 \mathrm{~cm}$, thus the relative difference in $R_{\max }-R_{\min }$ is approximately $2 \%$. We obtain similar results for passing orbits with different $E$ and $\Lambda$ and also for trapped orbits. These results for the particle orbits suggest that our approach will give a good approximation to the wave-particle dynamics. A detailed assessment of the impact of using the approximate approach on the particle orbits in Boozer coordinates will be pursued in future work.

\subsection{Calculations}

We now compute with HAGIS the resonant regions of fastion phase space for the $n=1$ TAE calculated in section 2 . The toroidal orbit frequency of ions is calculated using $\omega_{\phi}=\langle\dot{\phi}\rangle=\oint \dot{\phi} \mathrm{d} t / \oint \mathrm{d} t=\Delta \phi / \Delta t$, where the integral is performed over a single poloidal orbit. The poloidal orbit frequency is calculated as $\omega_{\theta}=2 \pi / \Delta t$. TRANSP simulations for MAST \#29221 show that the fast-ion pitch-angle distribution $f(\lambda)$ is approximately a Gaussian centred at $\lambda=\lambda_{0}=0.92$ with a width $\Delta \lambda=0.5$, where $\lambda \equiv v_{\|} / v$ is the pitch taken at the equatorial plane at the low-field side of the plasma cross section $\left(R>R_{\mathrm{mag}}\right)$. The focus of this paper is to illustrate the difference between the isotropic and anisotropic cases, so here we choose an idealised delta-function distribution, $f(\Lambda)=\delta(\Lambda)$, where $\Lambda$ is related to the pitch via $\Lambda=B_{0}\left(1-\lambda^{2}\right) / B$. Such a choice of distribution function is justified by the fact that most well-confined beam ions in MAST are in passing orbits.

Resonance maps for the $n=1$ TAE calculated in section 2 are graphed for the isotropic and anisotropic equilibria and wave frequencies in figure 9 as a function of $s^{2}$ and particle energy $E$. For both the isotropic and anisotropic cases the $l=0$ resonance occurs at $E \lesssim 3 \mathrm{keV}$ for all $s$. This resonance will not drive wave growth due to the strong energy-gradient damping. The broad $l=1$ resonance is significantly different between these cases; for a given particle energy the resonant region is located closer to the magnetic axis in the anisotropic case. No resonances for $l \geqslant 2$ are found in the energy range considered. (We note that these resonance maps are qualitatively similar to those in [31] for MAST \#29210, calculated for ions with $\Lambda=0.3$ resonating with a $n=1$ TAE.) 

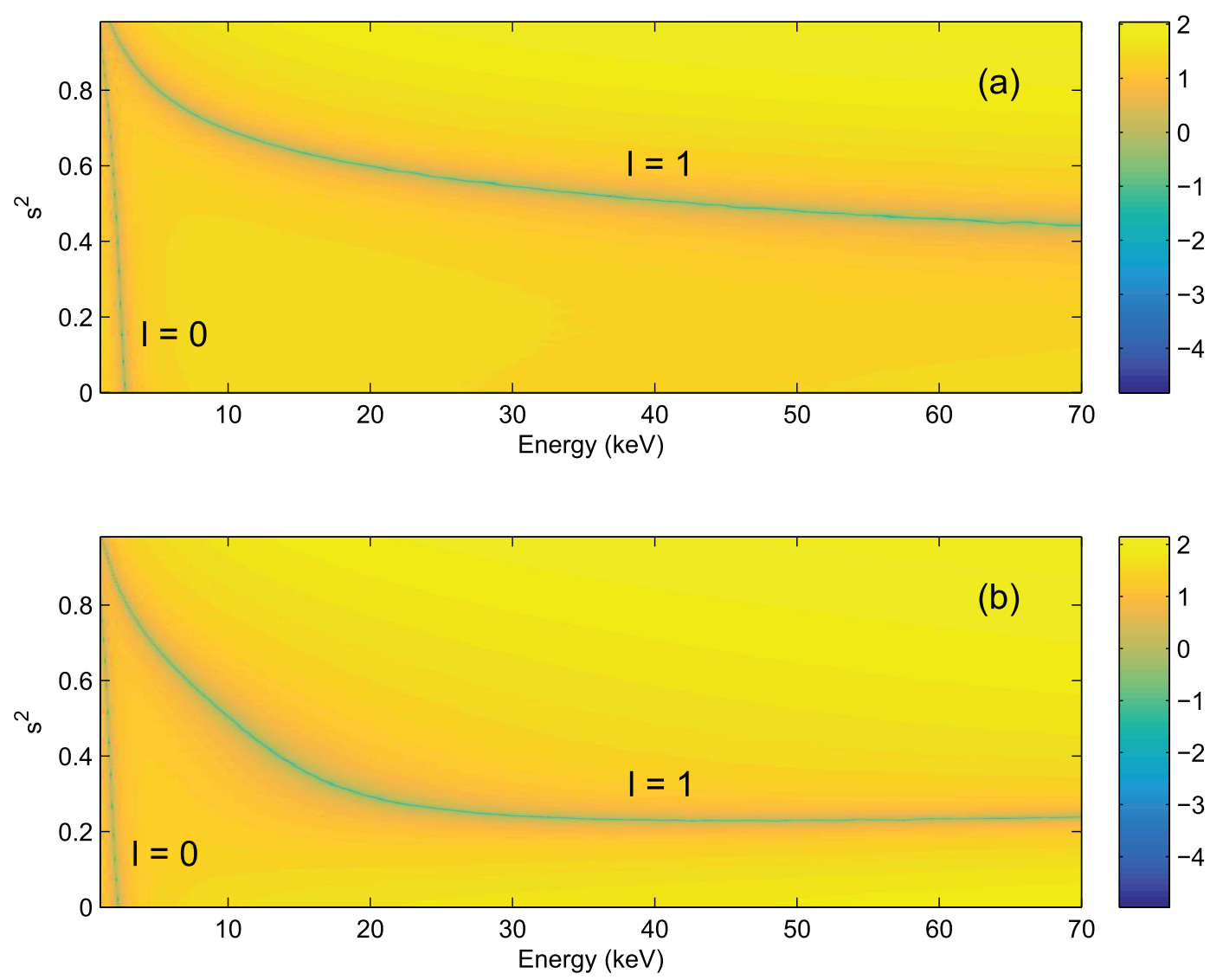

Figure 9. Resonance maps for ions with $\Lambda=0$ for $(a)$ isotropic equilibria and $n=1$ TAE of frequency $81.3 \mathrm{kHz}$, and $(b)$ anisotropic equilibria and $n=1 \mathrm{TAE}$ of frequency $88.9 \mathrm{kHz}$, with the bounce harmonics $l$ labeled. Colour indicates the value of $-\log _{10}\left(\sum_{l} 1 /\left|\Omega_{n, l}\right|\right)$, which is large and negative at resonances.

We now examine the difference in the wave amplitude evolution between the isotropic and anisotropic cases. The initial fast ion distribution function $f_{h}$ is chosen as a slowingdown distribution in energy and a Gaussian distribution in the radial coordinate $s$ (as in existing fast-ion physics studies on MAST [32, 33]):

$f_{h}(E, s, \Lambda)=\frac{C}{E^{3 / 2}+E_{c}^{3 / 2}} \operatorname{Erfc}\left[\frac{E-E_{0}}{\Delta E}\right] \exp \left[-\frac{s^{2}}{(\Delta s)^{2}}\right] \delta(\Lambda)$.

Using measured physical quantities at $t=0.190 \mathrm{~s}$ and fitting to TRANSP simulation data we estimate the values $E_{c}=16 \mathrm{keV}, E_{0}=65 \mathrm{keV}, \Delta E=1.5 \mathrm{keV}, \Delta s=0.5$, with the normalization constant $C$ chosen such that the on-axis fast ion density is $n_{h}=3.5 \times 10^{18} \mathrm{~m}^{-3}$. For our HAGIS simulations we choose 300000 markers to represent the fast ion population, and choose the integrator time step such that both the particle and wave integrators make 64 steps per wave period.

HAGIS calculations of the wave amplitude evolution are shown in figure 10 . We find that the linear growth rate for the isotropic case is $\gamma_{\text {iso }} / \omega_{\text {iso }}=2.08 \times 10^{-2}$, while for the anisotropic case $\gamma_{\text {aniso }} / \omega_{\text {aniso }}=2.81 \times 10^{-2}$. To explain this difference of $\approx 35 \%$ in the growth rates we first compare the drift-orbit widths and mode widths. We see from figure $5(a)$ that $q_{\text {aniso }}>q_{\text {iso }}$ for $s \gtrsim 0.25$. The resonant particles in the isotropic (anisotropic) case are located at $s \gtrsim 0.7$
( $s \gtrsim 0.5$ ) (see figure 9) and thus the safety factor is larger in the anisotropic case at the location of all resonant particles. The drift-orbit width of circulating particles $\Delta_{b}$ is dependent on the safety factor through $\Delta_{b} \approx q \rho_{\|}$(which was found to be a reasonable approximation for a spherical tokamak [34]), with $\rho_{\|}=v_{\|} / \Omega_{b}$ where $\Omega_{b}$ is the beam-ion gyrofrequency. The larger $q$ in the anisotropic case thus leads to a larger $\Delta_{b}$. Since the mode widths $\Delta_{m}$ of the dominant poloidal harmonics satisfy $\Delta_{m} \gtrsim \Delta_{b}$, the larger orbit widths in the anisotropic case are more comparable to the mode widths and are thus more favourable for mode drive. In addition, the $l=1$ resonance shown in figure 9 occurs at smaller $\psi$ for the anisotropic case; the radial gradient $\partial f / \partial \psi \propto \exp \left[-\left(\psi / \psi_{\text {edge }}\right) /(\Delta s)^{2}\right]$ is therefore larger at resonance in the anisotropic case, again leading to a larger growth rate through (2). HAGIS calculations for different fast-ion number densities $n_{h}$, shown in figure 11 , give larger linear growth rates in the anisotropic case than the isotropic case for each value of fast-ion number density.

Mode saturation is reached when the nonlinear bounce frequency of the trapped particles is similar to the linear growth rate, so that the fast depletion of wave energy by the trapped particles suppresses further wave growth [35]. The bounce frequency scales approximately as $\delta B^{1 / 2}$; mode saturation thus occurs when the field amplitude is sufficiently large such that $\omega_{b} \sim \gamma_{L}$, giving $\delta B_{\text {sat }} \propto \gamma_{L}^{2}$. Although $\gamma_{L}$ is larger in the anisotropic case, we find that the wave amplitude in 


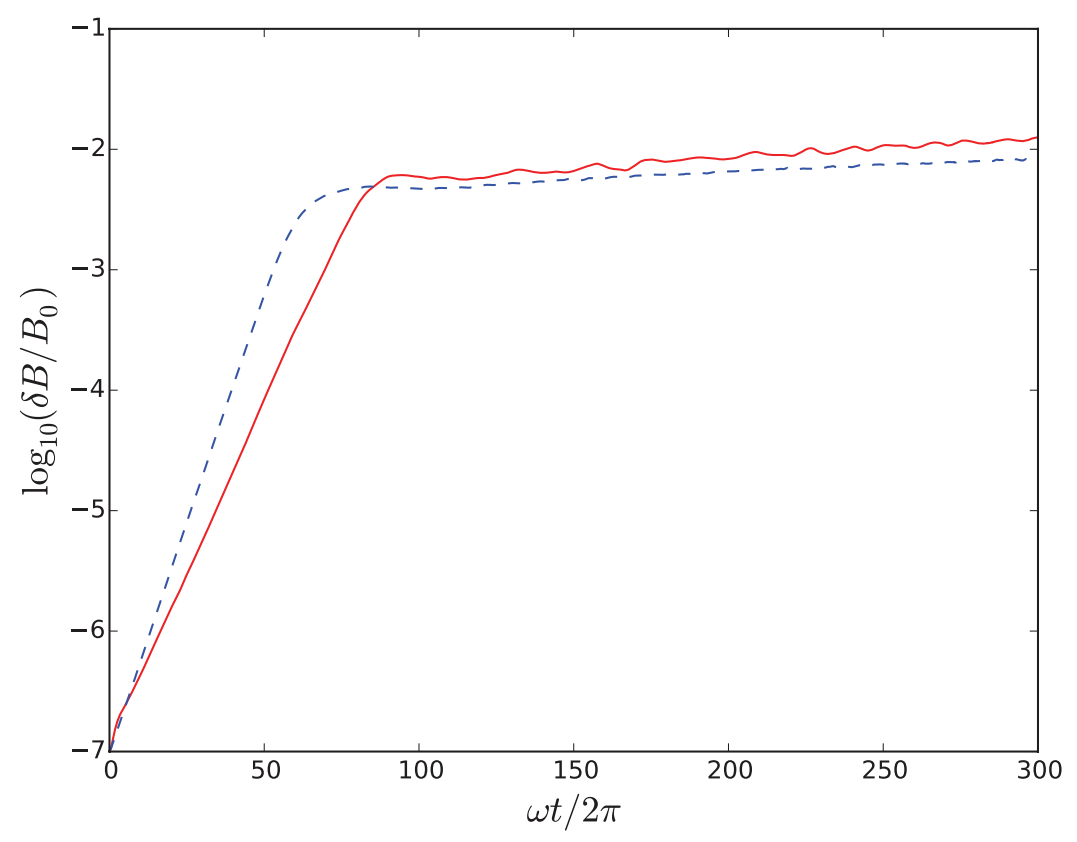

Figure 10. Wave amplitude $\delta B / B_{0}$ for the isotropic (red solid line) and anisotropic (blue dashed line) cases.

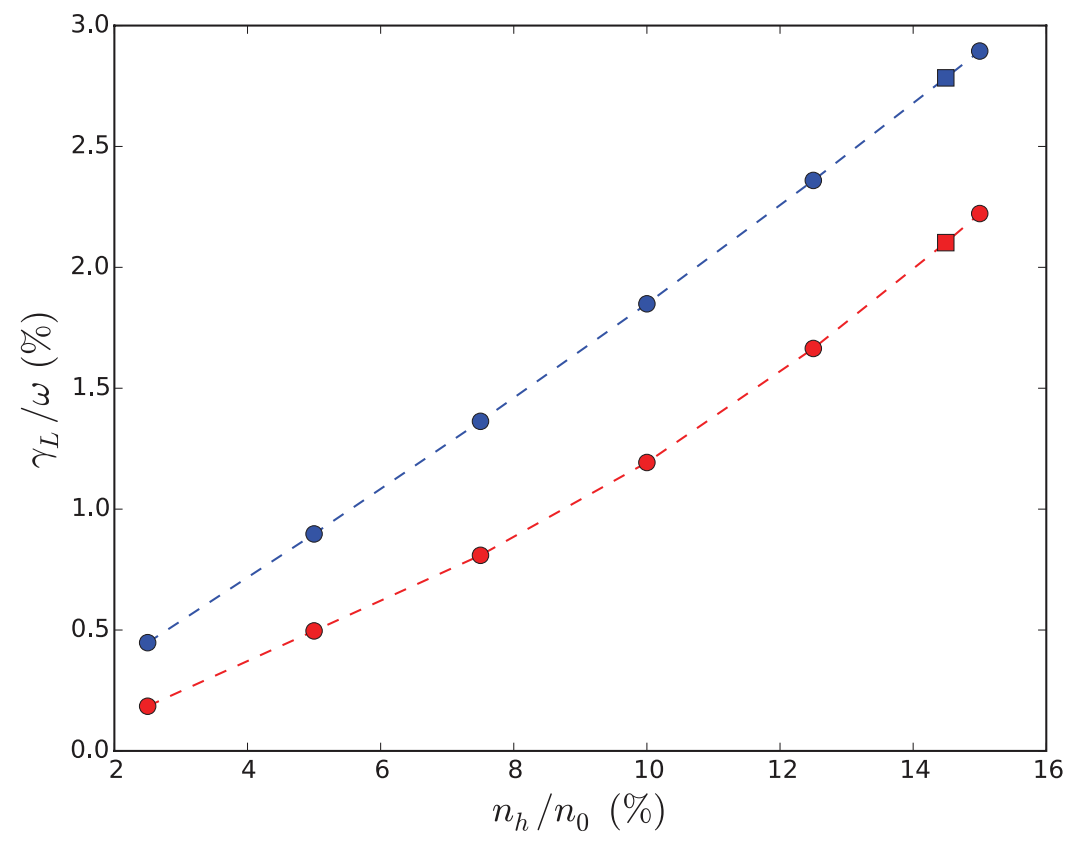

Figure 11. Linear growth rate $\gamma_{L} / \omega$ for different fast-ion density fractions $n_{h} / n_{0}$, with the isotropic case in red and the anisotropic case in blue. The square markers show the growth rates for the measured MAST \#29221 fast-ion density, $n_{h}=3.5 \times 10^{18} \mathrm{~m}^{-3}$.

the initial stage of saturation is $18 \%$ smaller in the anisotropic case $\left(\delta B / B_{0}=4.9 \times 10^{-3}\right)$ than in the isotropic case $\left(\delta B / B_{0}=6.0 \times 10^{-3}\right)$. To help explain this result we show in figure 12 a graph of $\log _{10}\left(\delta B / B_{0}\right)_{\text {sat }}$ versus $\log _{10}\left(\gamma_{L} / \omega\right)$, with the data points corresponding to the linear growth rates in figure 11. Both the isotropic and anisotropic cases are well fitted by the scaling $\delta B / B_{0}=D\left(\gamma_{L} / \omega\right)^{2}$, with $D$ a numerical constant of proportionality. We find in the isotropic case that $D=15.8$, while in the anisotropic case $D=6.8$. The smaller value of the constant $D$ in the anisotropic case results in a smaller saturation amplitude, despite its larger growth rate.
A possible explanation for the significant difference between the proportionality constants is that the bounce frequency is dependent on the equilibrium and mode structure. An analytical estimate of the saturation amplitude for a single poloidal harmonic of a TAE in a tokamak with circular flux surfaces gives a bounce frequency that depends on both the safety factor and magnetic shear (among other quantities) [36]. The safety factor and magnetic shear are significantly different between the isotropic and anisotropic cases (see figure 5), which may substantially account for the difference between the proportionality constants. 


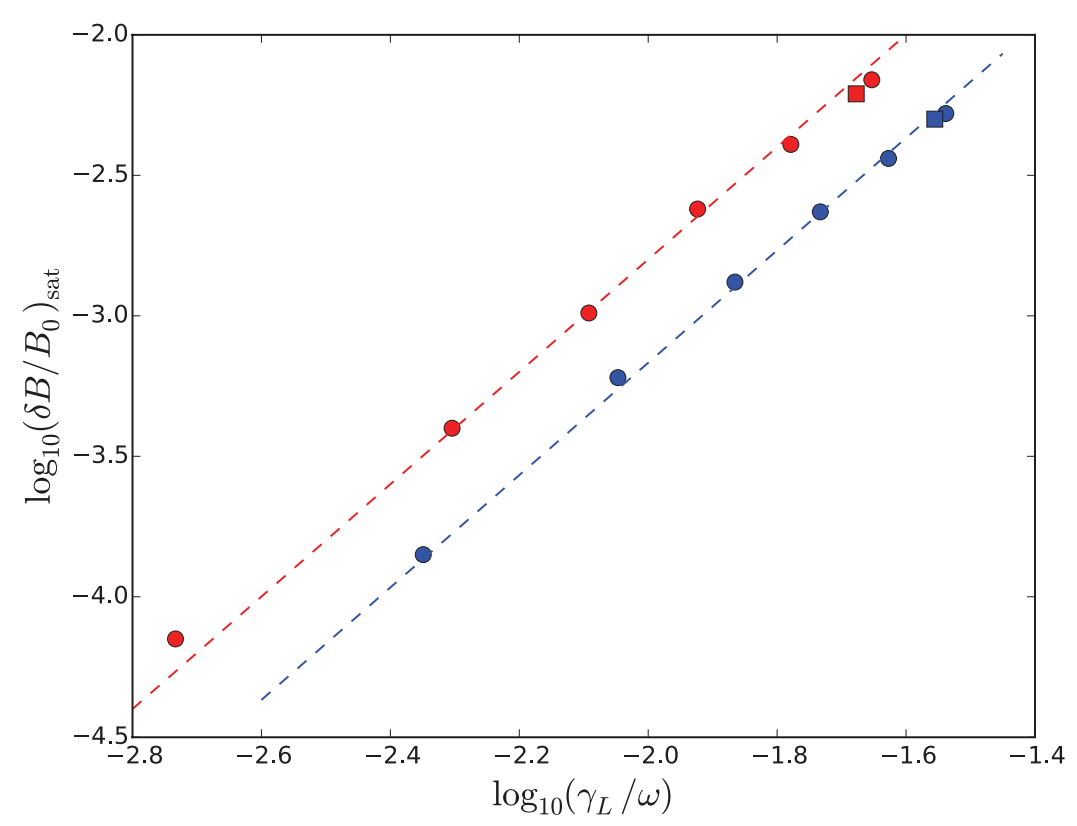

Figure 12. $\log _{10}\left(\delta B / B_{0}\right)_{\text {sat }}$ versus $\log _{10}\left(\gamma_{L} / \omega\right)$, with the isotropic case in red and the anisotropic case in blue. The square markers show the data points for the MAST \#29221 parameters, and the dashed lines show least squares fits to a linear function with slope 2.

\section{Conclusion}

We have analysed equilibrium and stability for MAST discharge \#29221 with the assumptions of both isotropic and anisotropic pressure. We find that quantities calculated under these two assumptions can be significantly different. The safety factor profile is qualitatively different between the two cases: in the isotropic case it is reversed shear, while in the anisotropic case it is normal shear. This difference leads to the TAE gap of the isotropic equilibrium being much narrower than that of the anisotropic equilibrium. The anisotropic $n=1$ TAE is found to have a larger radial width and slightly higher frequency than the isotropic TAE.

We find a significant modification to the resonant regions of phase space between the isotropic and anisotropic cases, with the anisotropic $l=1$ resonance shifted radially inward for given particle energy relative to the isotropic case. We also find a $35 \%$ larger linear growth rate in the anisotropic case compared to the isotropic case, while the anisotropic saturation amplitude is $18 \%$ smaller than the isotropic saturation amplitude. The linear growth rate for the anisotropic case is larger as the drift-orbit width is a closer match to the mode width, and the radial gradient of the distribution function at resonance is larger. Conversely, the saturation amplitude for the anisotropic case is slightly smaller despite the larger linear growth rate; this may be due to the differences in the safety factor and magnetic shear affecting the nonlinear bounce frequency. Further analysis, including studies of additional discharges and parameter scans of input quantities, will be needed to determine the likely and potential impact of pressure anisotropy on equilibrium and stability.

In these calculations we have neglected wave damping from the background plasma, as well as the effect of collisions on the fast ion distribution. Inclusion of these effects can modify the saturation amplitude and lead to various frequency sweeping behaviours depending on the relative magnitudes of the different collision terms [37]. The frequency sweeping and the mode turn-off occurring $2 \mathrm{~ms}$ after onset, observed in the spectrogram in figure 2, indicate that these effects are important in this MAST discharge. The impact of anisotropy on the frequency sweeping behaviour and saturation amplitude when damping and collisions are taken into account will be assessed in future work.

\section{Acknowledgments}

We thank Dr Owen Jones for access to TRANSP simulation results and Dr Ken McClements for helpful comments. This work was funded by the Australian Government through Australian Research Council grant DP140100790, the China Scholarship Council, and the Australian Institute of Nuclear Science and Engineering (AINSE) Postgraduate Research Award. This work was part-funded by the RCUK Energy Programme (under grant EP/I501045). The views and opinions expressed herein do not necessarily reflect those of the European Commission.

\section{References}

[1] Qu Z.S., Fitzgerald M. and Hole M.J. 2014 Plasma Phys. Control. Fusion $\mathbf{5 6} 075007$

[2] Zwingmann W., Eriksson L.G. and Stubber P. 2001 Plasma Phys. Control. Fusion 43 1441-56

[3] Hole M.J., von Nessi G., Fitzgerald M., McClements K.G., Svensson J. and The MAST Team 2011 Plasma Phys. Control. Fusion 53074021

[4] Hole M.J., von Nessi G., Fitzgerald M. and The MAST Team 2013 Plasma Phys. Control. Fusion 55014007 
[5] Layden B., Hole M.J. and Ridden-Harper R. 2015 Phys. Plasmas 22122513

[6] Cowley S.C., Kaw P.K., Kelly R.S. and Kulsrud R.M. 1991 Phys. Fluids B 32066

[7] Hsu S.C., Artun M. and Cowley S.C. 1996 Phys. Plasmas 3266

[8] Fitzgerald M., Sharapov S. and Hole M. 2011 Phys. Plasmas 18092508

[9] Pustovitov V.D. 2010 Plasma Phys. Control. Fusion 52065001

[10] Hole M.J. and Fitzgerald M. 2014 Plasma Phys. Control. Fusion 56053001

[11] Fitzgerald M., Appel L.C. and Hole M.J. 2013 Nucl. Fusion 113040

[12] Mikhailovskii A.B., Huysmans G.T.A., Kerner W.O.K. and Sharapov S.E. 1997 Plasma Phys. Rep. 23844

[13] Fitzgerald M., Hole M.J. and Qu Z.S. 2015 Plasma Phys. Control. Fusion 57025018

[14] Qu Z.S., Hole M.J. and Fitzgerald M. 2015 Plasma Phys. Control. Fusion 57095005

[15] Hole M.J., von Nessi G., Svensson J., Appel L.C. and The MAST Team 2011 Nucl. Fusion 51103005

[16] Keeling D.L. et al and The MAST Team 2015 Nucl. Fusion 55013021

[17] Huysmans G.T.A., Sharapov S.E., Mikhailovskii A.B. and Kerner W. 2001 Phys. Plasmas 84292

[18] Heidbrink W.W. 2008 Phys. Plasmas 15055501

[19] Coppi B. and Rewoldt G. 1976 Advances in Plasma Physics vol 6, ed A. Simon and W. B. Thompson (New York: Interscience) p 421

[20] Porcelli F., Stankiewicz R., Kerner W. and Berk H.L. 1994 Phys. Plasmas 1 470-80

[21] Fu G.Y. and Cheng C.Z. 1992 Phys. Fluids B 4 3722-34
[22] Breizman B.N. and Sharapov S.E. 1995 Plasma Phys. Control. Fusion 371057

[23] Berk H.L., Breizman B.N. and Ye H. 1992 Phys. Lett. A 162475

[24] Fülöp T., Lisak M., Kolesnichenko Y.I. and Anderson D. 1996 Plasma Phys. Control. Fusion $\mathbf{3 8} 811$

[25] Pinches S.D. et al 1998 Comput. Phys. Commun. 111 133-49

[26] Cooper G.A., Jucker M., Cooper W.A., Graves J.P. and Isaev M.Y. 2007 Phys. Plasmas 14102506

[27] Cooper W.A., Cooper G.A., Graves J.P. and Isaev M.Y. 2011 Phys. Plasmas 18052507

[28] Grad H. 1967 Proc. Symp. Appl. Math. 18162

[29] White R.B., Boozer A.H. and Hay R. 1982 Phys. Fluids 25575

[30] Hamilton B., McClements K.G., Fletcher L. and Thyagaraja A. 2003 Sol. Phys. 214 339-52

[31] Cecconello M. et al 2015 Plasma Phys. Control. Fusion 57014006

[32] Chapman I.T., De Bock M.F., Pinches S.D., Turnyanskiy M.R., the MAST Team, Igochine V.G., Maraschek M., Tardini G. and The ASDEX Upgrade Team 2009 Phys. Plasmas 16072506

[33] Chapman I.T., Hua M.D., Pinches S.D., Akers R.J., Field A.R., Graves J.P., Hastie R.J., Michael C.A. and The MAST Team 2010 Nucl. Fusion $\mathbf{5 0} 045007$

[34] McClements K.G., Gryaznevich M.P., Sharapov S.E., Akers R.J., Appel L.C., Counsell G.F., Roach C.M. and Majeski R. 1999 Plasma Phys. Control. Fusion 41661

[35] O'Neil T. 1965 Phys. Fluids 82255

[36] Wu Y., White R.B., Chen Y. and Rosenbluth M.N. 1995 Phys. Plasmas 24555

[37] Lilley M.K., Breizman B.N. and Sharapov S.E. 2010 Phys. Plasmas 17092305 